\title{
Durability of polyurethane - cement floors
}

\author{
Marek Maj ${ }^{1, *}$, Andrzej Ubysz $^{1}$ and Ashot Tamrazyan ${ }^{2}$ \\ ${ }^{1}$ Wroclaw University of Science and Technology, Wyb. Wyspiańskiego 27, 50-370 Wrocław, Poland \\ ${ }^{2}$ Moscow State University of Civil Engineering, Yaroslavskoe shosse, 26, Moscow, 129337, Russia
}

\begin{abstract}
The products covered by the common name polyurea were first developed and marketed in the United States in the 1980's In the first period polyurea was used as a protective coating of polyurethane foam used for thermal insulation of roofs, and in the 1990s many other uses were quickly disseminated. In Europe this technology appeared in the second half of the nineties and the market for polyurea applications started to develop very dynamically in the recent years. Polyurea coatings, due to their extremely versatile physical properties such as watertightness, extremely high wear resistance, resistance to chemical and mechanical stresses, high elasticity and stretchability as well as resistance to freezethaw cycles, are increasingly popular, and more and more frequently used in the broadly understood construction. As a rule, the growing demand for technology fosters its development and enhances the knowledge of those who use it, so that it is used for its intended purpose, while maintaining security and enforcement procedures. However, in the absence of such knowledge, misconceptions about the use of polyurea spray coating technologies are emerging, both at the design stage and during the execution phase.
\end{abstract}

\section{Introduction}

Industrial epoxy floors provide a protective layer for concrete floor slabs and must meet several stringent criteria. Floors particularly in the food industry as well as in the chemical industry are exposed to many destructive factors. Food processing often involves a combination of floor degrading agents creating a highly aggressive environment which conventional floors made of ceramic tiles or epoxy (see Fig. 3), polyurethane and polyurethane-cement resins are unable to withstand. Even though many of the epoxy floorings available today are resistant to individual burdens, such as abrasion, impacts, chemical aggression, high temperatures, damp floor bases, etc., only few of them can cope with the burdens if they occur simultaneously. For example, the structure of the material can be damaged as a result of a momentary change in some strength parameters due to high temperature (at which resin materials soften) and the simultaneous occurrence of mechanical loads. Similarly is in the case of the chemical aggression of most of the agents used in the food industry, which at elevated temperatures can be much more reactive and

\footnotetext{
* Corresponding author: marek.maj@pwr.edu.pl
} 
damaging than at ambient temperature. An example here is sodium hydroxide used in CIP rooms in food processing plants to disinfect and clean the installations, which at a $50 \%$ concentration at room temperature is moderately aggressive. But in the actual operational conditions it is used at a temperature of $60^{\circ} \mathrm{C}$. Then the reactivity level is so high that most of the epoxy flooring systems cannot withstand this chemical burden. One of the exceptions is the prototype of polyurethane-cement floors invented in the UK in 1969. Polyurea coatings are widely used also outside of the range presented in the article, wherever the following properties are expected of them, for example: application for external protective coatings, crack and fissures injection, re-profiling the structure, blocking of water leakage under pressure, replenishment of crushed edges, making a rounded surface on the junction of horizontal and vertical surfaces, pre-filling shrinking dilatation, gluing bands onto construction dilatations, different types of joining grounds.

\section{Chemical definition}

Polyurea is a substance obtained as a result of the chemical reaction of an isocyanate and a resin blend. Technically, this is a composite material processed by special spraying pumps using two-component and hot substances to form coatings or waterproofing insulations. The definition of Polyurea according to the PDA (Polyurea Development Association) is as follows:

Pure polyurea is a polymer derived from the reaction of a polyisocyanate component and a mixture of amino resin completed by amino groups. Polyurea is an elastomer obtained by the chemical reaction polyaddition of an aromatic or aliphatic isocyanate or an isocyanate prepolymer with a polyfunctional amine or a mixture of amines. It is generally a mixture of equal volumes (volume 100:100) [2]. In the aromatic system, isocyanate components are prepolymers based on methylenediphenyl diisocyanate (MDI), while in the aliphatic system - prepolymers of hexamethylene diisocyanate (HDI) or isophorone diisocyanate (IPDI), which form the rigid segment of the chain. It is the choice of isocyanate prepolymer that affects most properties of the finished polyurea product. When forming standard polyurea formulas methylenodiphenyl diisocyanate (MDI) is usually used, based on prepolymers containing $15 \%$ to $16 \%$ of isocyanate groups. (NCO is an isocyanate group of the formula $-\mathrm{N}=\mathrm{C}=\mathrm{O}$.) Polyisocyanates are isomers of cyanates containing (see Fig. 1) the $[-\mathrm{O}-\mathrm{C} \equiv \mathrm{N}]$ anion and fulminates containing anion $[-\mathrm{C} \equiv \mathrm{N}-\mathrm{O}]-$ ). Within this range of NCO groups content, a good compromise between the material viscosity and the reactivity of the system can be obtained. With lower values of isocyanate groups, prepolymers give the system more flexibility and less reactivity, but have higher viscosity. High viscosity affects the aggravation of the two components in the mixing chamber during application, leading to negative consequences for the quality of the material after bonding.

Aromatic isocyanate

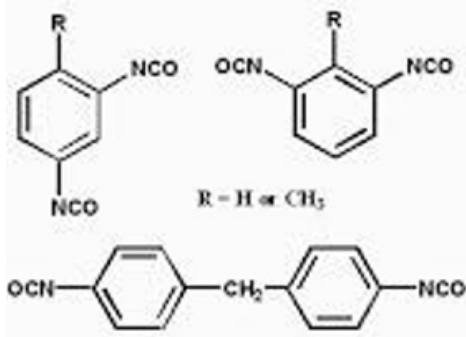

Aliphatic isocyanate

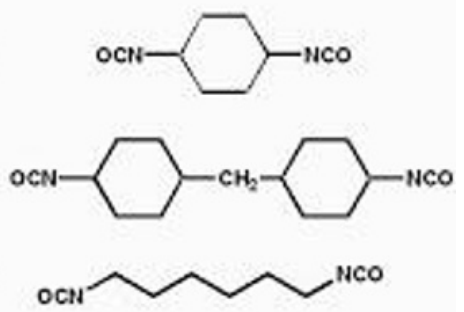

Fig. 1. Examples of aromatic or aliphatic isocyanate [2] 


\section{Material properties of polyurea coating after bonding}

The basic strength test in building mechanics is the dependence between tension and deformation. The concept of elasticity is close to that of construction materials and is most frequently understood as a deformation of interatomic bonds. The force acts on electrostatic bonds between atoms, causing the rise of the potential energy, and when it stops the atoms return to their original equilibrium state. In the case of materials considered to be so-called elastic, such as steel, these deformations are up to several tenths of percent. In the case of polyurea, the material also shows elastic properties (like most elastomers), but the nature of these deformations is different. These deformations result from the expansion of compact macromolecular chains. This straightening of the chains is not related to the mutual movement of atoms, so there is no "elongation" of the bonds between them. Elongation of a material, caused by, for example, the action of force, leads to decreased entropy. It is accompanied by, among others, a heat emission effect. When the force stops working, the entropy rises (the material absorbs heat) and the material returns to its original length. In this case the elongation of the material can be even the multiples of the original dimensions. However, after relieving, most materials exhibit relatively large permanent deformations and the "return" time to the original dimensions is much slower. This is the effect of the property called viscoelasticity.

The above phenomena, despite the similar description, are of a different nature, and therefore the concepts of elasticity and flexibility are distinguished. For some applications, the concept of flexibility, which, although does not come under such simple physical dependencies, is used to better characterize the properties of the material. Elasticity is an important feature of the material, in terms of its flexibility, which is particularly important in the application of the material on a soft thermal insulation, which can bend under load during the exploitation (e.g. mineral wool on a roof covered with a spray coating). The use of polyurea coating greatly extends the service life of the diffusers, which, without protection, are cracked by vibration and cyclical deformation caused by the rush of air being thrown out of the hall. The connection between chemical resistance of the material and its elasticity is very closed. Materials with high density of molecular bonds are difficult to destroy during chemical loading, demonstrating good resistance to such interactions (see Fig. 2). However, these bonds have very short gaps between the "nodes" (bindings), which hinders any movement of individual molecules (molecules), which in turn makes the material very rigid.

a)

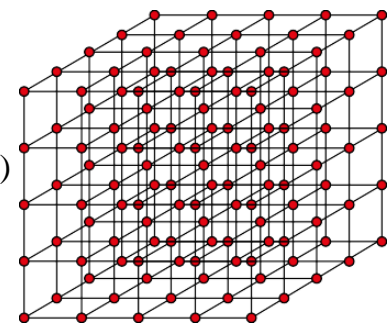

b)

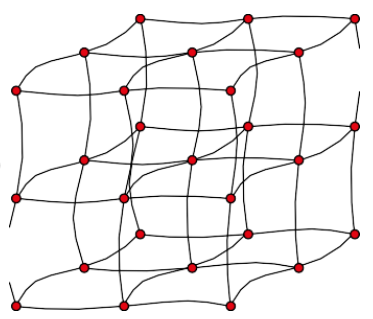

c)



Fig. 2. a) A model of polymer bonds grid in rigid material [2]

b) A model of polymer bonds grid in resilient (elastic) material.

c) A model of damage to a material with low density of polymer bonds.

Rigid materials used as impermeable protection against penetration of harmful substances into groundwater as a rule do not meet the assumed requirements because they tend to form micro cracks and scratches due to deformation of the concrete structure caused by temperature changes. All cracks and fissures allow aggressive substances to enter the 
concrete causing degradation and peeling of the coating. As a consequence they allow these aggressive and harmful substances to enter groundwater. Materials with low density of polymeric bonds leave "great freedom" of moving for individual particles of material $[1,2]$. This results in high elasticity of the coating, which, along with the work of the substrate (e.g. alternating dilating and shrinking under varying ambient temperatures) will also stretch and shrink without cracking [5], [6], [7]. A coating with such properties will effectively protect a concrete structure against degradation and possible penetration of harmful substances into the groundwater. However, the lower the crosslinking density, the easier it is to destroy less protected molecules by "a chemical attack". To show the cause, this mechanism can be compared to filtering water or air - the finer the sieve (the filter with finer mesh), the more it will stop the pollution. Similarly, the thicker the cross-linking of chemical bonds in the coating material, the more difficult it is to destroy its structure, because there is more single protection against the destruction of its structure. By providing chemical protection, we cannot choose between the tightness resulting from the material flexibility and its chemical resistance, since both of these parameters are equally important to provide a lasting protection.

\section{Resistance of floors to various impacts}

It must be underlined that most of the mechanical impact on industrial floors are somehow related to non-uniform geotechnical conditions under the floor slab. The importance of proper evaluation of ground properties [3] and some examples of slab testing [4] can be found in many references. The authors of the current study focus on impacts on cover layer.

\subsection{Resistance to chemical effects.}

It is often the case that a combination of chemical substances acts on floors much more aggressively than the same substances acting separately. For example, in the food industry many different chemical substances, which are not present in the final products, are used in the technological processes. The substances are:

- $50 \%$ acetic acid, widely used as spirit vinegar in the food industry, particularly in the production of soups, sauces and vegetable preserves; acetic acid used in different concentrations at a temperature of $60^{\circ} \mathrm{C}$ in the production of various dairy products;

- $100 \%$ oleic acid at a temperature of $60^{\circ} \mathrm{C}$, representing organic acids forming as a result of the oxidation of vegetable and animal fats widely used in the food industry;

- concentrated citric acid, occurring, i.a., in citrus fruits, representing a wider range of fruit acids which very quickly can degrade epoxy floors; wines and hand disinfectants;

- $50 \%$ sodium hydroxide at a temperature of $60^{\circ} \mathrm{C}$, used as a cleansing agent;

- $30 \%$ nitric acid, used as a cleansing agent; $15 \%$ sodium hypochlorite - a disinfectant.

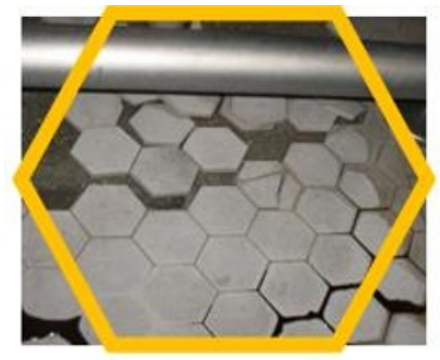

Fig. 3. Loose and shifted tiles in dairy floor

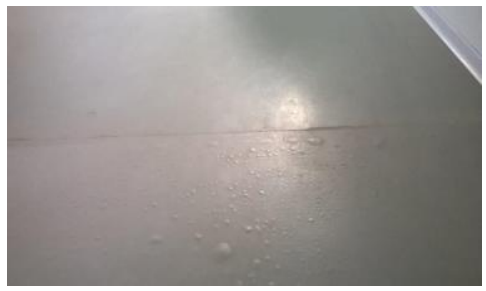

Fig. 4. Difference between two different polyurethane-cement floorings laid on the same floor 
As regards chemical burdens, one of the most harsh environments for floors in the food industry is milk processing. For this reason the most commonly used type of flooring in dairies are chemical resistant tiles. But in such a floor, even if grouted with epoxy resin, the grout joints and the tile adhesive dissolve and the tiles become loose under the action of caustic substances (see Fig. 3). As a result, the tiles are susceptible to cracking under concentrated mechanical loads.

\subsection{Resistance to mechanical impacts}

Besides the required high chemical resistance, industrial floors should show high impact strength. Strong mechanical impacts vary in their form and magnitude. They occur, for example, when meat hooks are dropped, steel hose clamps fall down, steel barrels fall out of the production line, tools and lids are dropped during maintenance and so on. A strong and durable floor characterized by a high energy absorption capacity minimizes damage and prevents cracking, the penetration of chemicals through the cracks into the structure of the concrete under the epoxy floor and the degradation of the latter from below. Such a floor ensures safety and a long floor service life without renovation.

Contrary to appearances, it is not high hardness which can ensure floor resistance to impacts by sharp objects, but a low modulus of elasticity (in the order of 3000-3500 MPa) thanks to which the structure of the material is elastic and compressible, whereby the material does not spall and the floor can absorb much more energy than concrete with a strength of $50 \mathrm{MPa}$. Only some of the polyurethane-cement floorings, which (as opposed to epoxy floors or floors made of chemical resistant tiles) owing to their high compressibility under impact dissipate energy and do not crack or debond from the base, have the above properties.

\subsection{Resistance to mechanical impacts}

In many industries high temperatures originating from both production and clean up processes (e.g. cleaning tiles with steam) act on the floor. The action of high temperatures can momentarily weaken the resistance of the floor to mechanical loads, which often occur simultaneously. An example here can be the bottling plant areas in which the inside and outside surfaces of kegs are cleaned and sterilized and subsequently the kegs are filled with bear, labelled and rolled on the floor (see Fig. 5).



Fig. 5. Transport of kegs of beer on hot floor



Fig. 6. Thermal and chemical burdens acting on floor in technological cycle

As many as 1000 kegs are cleaned and filled per hour. It is not a rare occurrence that a filled keg weighing $72 \mathrm{~kg}$ falls down from the production line and hits the floor. The latter is constantly exposed to the action of detergents and disinfectants up to $80^{\circ} \mathrm{C}$ hot, whose acidity and alkalinity range from $1 \mathrm{pH}$ (a strongly acid environment) to $13.8 \mathrm{pH}$ (a strongly alkaline environment). 
In such situations a special floor resistant to many different factors is required to ensure resistance (without cracking and debonding) to strong impacts as well as resistance to a wide range of chemical agents. Temperatures affect the floor in two ways: 1) soften the floor material and 2) generate thermal stresses due to the thermal expansion of the material. This to some degree also applies to the surface of the contact between the concrete and the floor since the thermal expansion coefficients of the two materials are different. Then shear stresses occur at the bond between the epoxy floor and the concrete underlayment. As a result, epoxy floors debond from the concrete base and the floor in the loose areas is easily damaged under mechanical loads. This often occurs in various industrial plants (see Fig. 3).

Among the currently available polyurethane-cement floorings there is one whose softening temperature is as high as $\sim 130^{\circ} \mathrm{C}$ and whose thermal expansion coefficient is close to that of concrete, i.e. $4 \times 10^{-5 o} \mathrm{C}$-1, whereby it can withstand extreme thermal shocks see Fig. 6).

Most epoxy floors lose their hardness at a temperature of $60^{\circ} \mathrm{C}$ (or not much higher), whereas floors based on polyurethane-cement resins maintain their high strength and hardness until the temperature of $130^{\circ} \mathrm{C}$ is exceeded. However, high temperature has no such a marked effect on the durability of floors as a temperature gradient occurring over a short time (a thermal shock) does. Due to considerable local stress gradients a thermal shock can cause damage to many kinds of flooring. A thermal shock occurs as a result of a rapid change in temperature, for example, in a place where a hot liquid has leaked or spilled onto the floor under a process line operating at high temperatures.

\section{Use and durability of polyurethane-cement floors}

Cement polyurethane industrial flooring is available in many thickness ranges, starting with a $4 \mathrm{~mm}$ thick floor that is fully functional up to $70^{\circ} \mathrm{C}$ and ending with a $12 \mathrm{~mm}$ thick floor that is most suitable for the most extreme environments with instantaneous thermal load up to $150^{\circ} \mathrm{C}$. The greater thickness ensures better thermal insulation of the insulation on the contact surface between the polyurethane floor and the concrete base, thus preventing the loads caused by the thermal shock. It is natural that a properly designed ceiling base is required in extreme thermal shock conditions. In particular, the potentially large thermal deformations of the base must be taken into account.

There is no simple test that would confirm that the floor will withstand the thermal shock repeated in an industrial environment for many years. This is due to the varying quality of the substrate and various projects, especially in old buildings where the floors have been renovated and renovated many times. Without many years of observations being carried out on the spot for many years, it is difficult to predict how the floors will behave in such conditions. This may be the main argument when choosing a cement and polyurethane flooring system.

The most difficult conditions resulting from the coincidence of high temperatures as well as mechanical and chemical loads occur in the areas in which racks or baskets on hot steel wheels move, e.g. after removal from furnaces or autoclaves. Previous observations indicate that only floors based on cemented polyurethane reinforced resin (FI floors) work well in such extreme conditions. This type of floor can also withstand extreme mechanical loads, such as shadowed or steel motion of wheeled vehicles and moving steel containers or steel loaders. 


\section{Hygienic and sanitary requirements which floors must meet}

Sometimes hygiene is an important consideration in the selection of epoxy flooring systems for industrial plants. Many parts and devices intended for the food industry are made of stainless steel, which makes it easier to maintain the required level of sterility of the equipment. Similar requirements apply to floors, which should be non-absorbing, easy to clean and lend themselves to antibacterial, chemical or radiational treatment.

Good quality polyurethane-cement floors are tested in this regard and the results of independent tests confirm that they can be kept as bacterially clean as stainless steel. After their application many resinous materials require a waiting period in order, for example, to eliminate the risk of contamination of food products. Therefore prior to deciding on a particular flooring it is worth checking if the material meets this requirement.

The polyurethane-cement floor systems available on the market are not only free from any substances which would pose an air contamination hazard during floor laying, but also are free from noxious odours, which is an important consideration in the case of renovations, alterations and extensions. Thanks to this they are allowed to be laid in a production area in which production is being conducted. Naturally, the above aspects should be confirmed by proper certificates.

Nevertheless, one should take precautions that such an extension or alteration of the production area does not cause any unnecessary disturbances and that polyurethane-cement materials characterized by high tolerance to floor base dampness (without it being necessary to determine the percentage moisture content) are used. Otherwise, if not seasoned for at least 28 days until the moisture content of less than $4 \%$ is achieved, the floors will deboned from the concrete base. The Figure 4 shows such a difference between two polyurethane-cement flooring systems laid on the same base at the same time. The photograph was taken after six months since the application of the floorings. In one of the floorings one can clearly see blisters caused by diffusion pressure, whereby the epoxy flooring debonds from the surface of the concrete.

\section{Polyurethane-cement floor installation time}

In investment processes a major consideration is how quickly a given material begins to bond and reaches full strength. Polyurethane-cement materials are characterized by a short bonding time which is an important consideration when a given room has to be put out of use for floor laying.

The costs due to the loss of production during such works often can exceed to the costs of the floor itself. For economic reasons alone it is worth taking a look at various materials since the differences in this respect can be very large. Bonding time since the laying of a floor coating ranges from 5 hours when Fast systems are used to as many as 72 hours for other floorings based on polyurethane-cement resins.

\section{Conclusions}

Presented applications shows that polyurea coats are suitable for cover and strengthen many materials and concrete especially. Taking into account the costs of materials, time and technology, it can be said that polyurea coatings can be one of the most commonly used technologies in a short time.

Only a small class of floors from among all epoxy floors are able to withstand such a wide range of chemicals and thermal loads without losing their strength. An example here 
is a polyurethane-cement flooring system manufactured by ICI in Britain, whose chemical resistance is equal to that of special vinyl-ester systems, acid resistant facing brick and chemical resistant tiles laid and grouted using an adhesive based on furan resins. There are only few polyurethane-cement technologies which make it possible to obtain a material whose softening temperature is as high as $+130^{\circ} \mathrm{C}$ and which at the same time has a thermal expansion coefficient close to that of concrete, i.e. $4 \times 10-5^{\circ} \mathrm{C}-1$, whereby it can withstand extreme thermal shocks. Since such floors are exceptionally dense and completely non-absorbing it can be assumed that without any bactericidal additions they make any development of bacteria on their surface practically impossible. Thus, in many cases, using such flooring systems one does not need to worry about undesirable effects of such additions.

This paper has been written as part of the research project: "Industrialized construction process (Construction 4.0). Technological and methodological conditions of application of selected composite elements in civil engineering". The project is being carried out jointly with Peoples' Friendship University of Russia in Moscow. Research project PWr-RUDN 2017 no. 45WB / 0001/17 Industrialized Construction Process.

\section{References}

1. L. Czarnecki, J.R. Clitton, W. Gładkowska, Problem of compatibility of polymer mortars and cement concrete system, International Colloquium Materials and Restorasion, Esslingen, 964-971 (1992)

2. J. Banera, M. Maj, A. Ubysz, Powłoki Polimocznikowe w Budownictwie, BASF, DTP:D-CONCEPT, Grupa MD A 2017

3. D. Sobala, J. Rybak, Role to be played by independent geotechnical supervision in the foundation for bridge construction, IOP Conf. Series - Materials Science and Engineering, 245, 022073 (2017)

4. J. Vlcek, M. Drusa, W. Scherfel and B. Sedlar, Experimental Investigation of Properties of Foam Concrete for Industrial Floors in Testing Field, IOP Conf. Series Earth Environ. Sci., 95 (2), 022049 (2017)

5. J. Kokowska, Badania pokrywania rys w podłożu betonowym przez powłoki polimerowe, Building Research Institute -Quartely, 3 (151), (2009)

6. A. M. Brandt, Cement based composites. Materials, mechanical properties, and performance, 2ED Taylor\&Francis Group (2009)

7. EN 1062-7 2004 Paints and varnishes; Coating materials and coating systems for exterior masonry and concrete - Part 7: Determination of crack bridging properties 